\title{
Molecular characterization of cathepsin $L$ cDNA and its expression during oogenesis and embryogenesis in the oriental river prawn Macrobrachium nipponense (Palaemonidae)
}

\author{
W. Zhao ${ }^{1,2}$, L. Chen ${ }^{1}$, F. Zhang ${ }^{1}$, P. Wu ${ }^{1}$, E. Li ${ }^{1}$ and J. Qin ${ }^{3}$ \\ ${ }^{1}$ College of Life Science, East China Normal University, Shanghai, China \\ ${ }^{2}$ School of Chemical and Biological Engineering, \\ Yancheng Institute of Technology, Yancheng, China \\ ${ }^{3}$ School of Biological Sciences, Flinders University, Adelaide, Australia \\ Corresponding author: L. Chen \\ E-mail: lqchen@bio.ecnu.edu.cn
}

Genet. Mol. Res. 12 (4): 5215-5225 (2013)

Received March 27, 2013

Accepted August 19, 2013

Published October 30, 2013

DOI http://dx.doi.org/10.4238/2013.October.30.6

\begin{abstract}
We identified the cDNA sequence of cathepsin L (CatL) in Macrobrachium nipponense, designated as MnCatL, for the first time. The MnCatL cDNA, isolated from the ovary, was 1710 $\mathrm{bp}$ in length, containing a 31-bp 5'-untranslated region, a 650-bp 3 '-untranslated region, and an open reading frame of $1029 \mathrm{bp}$, encoding a 342-amino acid polypeptide with a predicted molecular mass of 37.7 $\mathrm{kDa}$. The polypeptide is composed of an 18-amino acid signal peptide, a 106-amino acid propeptide and a 218-amino acid mature peptide. MnCatL mRNA was detected in all tissues that we examined, including the thoracic ganglia, heart, muscle, intestine, hemocytes, ovary, testis, gills, and hepatopancreas. MnCatL expression reached a maximum value in both hepatopancreas and ovaries at the later stages of vitellogenesis, suggesting that MnCatL is involved in ovarian maturation of the oriental river prawn. During embryogenesis, MnCatL expression decreased as
\end{abstract}


the embryo developed. The expression of MnCatL in the ovary and embryo suggest that MnCatL plays an important role in the uptake of vitellogenin and yolk protein, which are deposited in the oocyte for ovary maturation and embryo development, during oogenesis and embryogenesis of M. nipponense.

Key words: Cathepsin L; Macrobrachium nipponense; Ovarian maturation; Embryo development; Yolk protein degradation

\section{INTRODUCTION}

Vitellogenin (VTG) is a large multi-domain apolipoprotein that serves as the precursor of major egg yolk proteins in oviparous animals (Wallace, 1985; Chen et al., 1997). Under a weak acidic condition maintained by vacuolar proton-ATPase, VTG is cleaved into yolk (Fagotto and Maxfield, 1994) and then hydrolyzed during later stages in oocyte development. The uptake of VTG occurs during embryogenesis to provide nutrition for embryo development.

VTG can be degraded in the lysosome through endocytosis. The smaller molecules produced by endocytosis are subsequently exported from the lysosome by a vesicle-mediated process to yolk globules/granules to support vitellogenesis (Carnevali et al., 2006). The most abundant cysteine protease involved in vitellogenesis is cathepsin $\mathrm{L}(\mathrm{CatL})$, which is believed to degrade proteins in the lysosome of vertebrates. For instance, in the ovary of sea bream (Sparus aurata), the high level of CatL can completely digest yolk proteins such as lipovitellin (Carnevali et al., 1999). Similar functions of CatL in vitellogenesis were also found in rainbow trout Oncorhynchus mykiss (Kwon et al., 2001) and Danio rerio (Carnevali et al., 2006). Most studies on CatL in arthropods have focused on the function of CatL in immunity (Ren et al., 2010; Zhang et al., 2010b) and digestion (Hu and Leung, 2007; Soares-Costa et al., 2011), but little is known of its role in VTG utilization. Current evidence in Metapenaeus ensis (Crustacean) suggests that the ovary is the only non-alimentary organ showing CatL expression (Hu and Leung, 2004). In addition, a CatL-like cysteine proteinase is present in the oocyte of Sitophilus zeamais (Matsumoto et al., 1997) and silkmoth Bombyx mori (Yamamoto et al., 1994). Furthermore, CatL exists in the yolk as a proenzyme, which can be activated by partial proteolysis at low $\mathrm{pH}$ in Ornithodoros moubata (Fagotto, 1990a,b). These findings led us to believe that CatL or CatL-like substances in the ovary in arthropods may be functionally important in reproductive physiology such as degradation of vitellogenin and yolk protein. In addition, CatL also exists in the hepatopancreas and at a level 10 times higher than in other tissues in crustaceans (Hu and Leung, 2004; Li et al., 2010). In crustaceans, hepatopancreas is not only involved in food digestion but also in the synthesis and degradation of VTG (Meusy and Payen, 1988). Therefore, it is important to explore the patterns of CatL expression in the hepatopancreas and ovaries to better understand the function of Cat $\mathrm{L}$ in crustacean reproduction.

In this study, we used the oriental river prawn Macrobrachium nipponense as a model species of crustaceans to examine VTG synthesis in both the ovary and hepatopancreas. We first cloned the CatL gene named MnCatL from the ovary tissue and then examined the expression of MnCatL in the hepatopancreas and ovary during oogenesis and embryogenesis. Particularly, we also investigated the effect of CatL on the degradation of VTG and yolk proteins to determine its role in crustacean reproduction. 


\section{MATERIAL AND METHODS}

\section{Animals}

Female oriental river prawns, $M$. nipponense (0.6-2.1 g) at various ovarian maturation stages were obtained from a local seafood market in Shanghai, China. The prawns were cultivated in freshwater for 10 days at ambient temperature until tissue extraction. Ovarian and embryonic developmental stages were identified according to Zhao et al. (2011) and Zhang et al. (2010a), respectively. The tissues and embryos analyzed were dissected, rinsed with phosphate-buffered saline, snap-frozen in liquid nitrogen, and stored at $-80^{\circ} \mathrm{C}$ until extraction. All tissues tested were from female prawns except that the male hemocytes (HAM), male hepatopancreas (HPM) and testis were from male prawns.

\section{Total RNA extraction and reverse transcription}

All tissues and embryos analyzed were homogenized in Unizol Reagent (Biostar, Shanghai, China), and total RNA was extracted following the manufacturer instructions. The purity of RNA was measured by $\mathrm{OD}_{260} / \mathrm{OD}_{280}$ values. cDNA was synthesized from $5 \mu \mathrm{g}$ total RNA by the Takara PrimerScript ${ }^{\mathrm{TM}}$ First-Strand cDNA synthesis kit (TaKaRa, Dalian, China) according to the manufacturer protocol.

\section{Cloning of MnCatL cDNA}

Initially, PCR was performed using the cDNA prepared above as template. The partial fragment of MnCatL cDNA was obtained by specific primer CLF (5'-AAAAGTGTTGACTG GAGGGA-3') and CLR (5'-CAGCATTTGCAGGATTGTAC-3') based on an initial expressed sequence tag isolated from an ovary cDNA library (Wu et al., 2009).

MnCatL was cloned by the method of rapid amplification of the cDNA ends (RACE). The cDNA for 5'-RACE and 3'-RACE was synthesized according to the manufacturer protocol using the Smart Race kit (Clontech, Palo Alto, CA, USA) and 3'-RACE System (Invitrogen, Carlsbad, CA, USA), respectively. A total of $5 \mu \mathrm{g}$ RNA was reverse transcribed using MMLV reverse transcriptase (TaKaRa, Dalian, China) with the adapter primer (AP: 5'-GGCCACGCGT CGACTAGTACTTTTTTTTTTTTTTTTT-3') for 3'-RACE and the 5'-CDS primer A (5'-(T)25 $\mathrm{VN}-3$ ', N = A, C, G, or T; V = A, G, or C) for 5'-RACE to obtain the first-strand cDNA. The 5'and 3'-regions of MnCatL were amplified by nested PCR using two 5'-RACE primers (5'CL1: 5'-CGGCATGGTTCGTCCTCTGCTTC-3', 5'CL2: 5'-GCATGGTTCGTCCTCTGCTTCAT-3') and two 3'-RACE primers (3'CL1: 5'-CCCTTATGAAGCAGAGGACGAAC-3', 3'CL2: 5'-ACGAACCATGCCGGTACAATCCT-3'), which were designed from the known nucleotide sequence of the MnCatL cDNA fragment. The 5'CL1 and $3^{\prime} \mathrm{CL} 1$ were used as the first amplification primers, and $5^{\prime} \mathrm{CL} 2$ and $3^{\prime} \mathrm{CL} 2$ were used as the nested primers.

The homology search for the nucleotide and protein sequences was performed using the BLAST algorithm at NCBI (http://www.ncbi.nlm.nih.gov/). The deduced amino acid sequence was analyzed with the Expert Protein Analysis System (http://www.expasy.org/). The full-length multiple alignment of the MnCatL amino acid sequence was compared with CatL of other organisms. Amino acid sequences from various species retrieved from the NCBI Gen- 
Bank were analyzed using the ClustalW Multiple Alignment program (http://www.ebi.ac.uk/ clustalw/) and GeneDoc program.

\section{Quantitative real-time PCR (qPCR)}

The expression of MnCatL mRNA was demonstrated by a SYBR green real-time qPCR analysis in an ABI StepOne plus Sequence Detection System (ABI). The RNA obtained from all tissues and embryos used for cDNA synthesis was digested with RNase-free DNase I to eliminate possible genomic DNA contamination.

The qPCR amplifications were carried out in a total volume of $20 \mu \mathrm{L}$, containing 10.0 $\mu \mathrm{L} 2 \mathrm{X}$ SYBR Premix Ex Taq (TaKaRa, Dalian, China), $2.0 \mu \mathrm{L}$ cDNA diluted with DEPC-water, $0.4 \mu \mathrm{L} 50 X$ ROX Reference Dye and $0.5 \mu \mathrm{L}$ (each) qCLF and qCLR primers $(2 \mathrm{mM})$, which were 5'-GTGGTAATGGAGGAGTGGGAG-3' and 5'-TAGAGCTTGTTGTGGGCAGC-3', respectively. Before the formal test, the specificity of the primers was determined using the melting curve method. The standard curves for MnCatL (efficient: 0.925; slope: -3.217; intercept: $31.919 ; \mathrm{y}=-3.517 \log \mathrm{x}+31.919 \mathrm{R}^{2}=0.993$ ) and $\beta$-actin (efficient: 0.914; slope: -3.546; intercept: $\left.35.607 ; y=-3.546 \log x+35.607 \mathrm{R}^{2}=0.996\right)$ were derived, suggesting that the primers designed were highly specific for the target sequence. Amplification of $\beta$-actin was carried out in the same sample as an internal reference (Zhang et al., 2010a; Zhao et al., 2011). The qPCR temperature profile was $95^{\circ} \mathrm{C}$ for $10 \mathrm{~s}$ followed by 40 cycles of $95^{\circ} \mathrm{C}$ for $10 \mathrm{~s}$, and $60^{\circ} \mathrm{C}$ for $1 \mathrm{~min}$. DEPC-water for the replacement of template was used as a negative control.

\section{Data analysis}

The MnCatL and $\beta$-actin standard curves were constructed with 10 -fold serial diluted cDNA templates. The concentration of cDNA in each sample was calculated from the standard curve. The amount of MnCatL mRNA was normalized to the $\beta$-actin transcript level. All data are reported as means $\pm \mathrm{SE}$ (standard error). The results were subjected to one-way ANOVA by SPSS17.0, and the level of significant difference was set at $\mathrm{P}<0.05$.

\section{RESULTS}

\section{Characterization of MnCatL cDNA}

The full length of the cDNA of MnCatL gene was obtained in this study and deposited in GenBank (accession No. HM134078). The nucleotide sequence and the deduced amino acid sequence are shown in Figure 1. The MnCatL gene included a 31-bp 5'-terminal untranslated region (UTR), a 650-bp 3'-UTR and a 1029-bp open reading frame, encoding a 342-amino acid protein polypeptide composed of an 18-amino acid signal peptide, a 106-amino acid propeptide and a 218-amino acid mature peptide, with predicted molecular mass of $37.7 \mathrm{kDa}$. In the 3'-UTR, there was one 25-bp poly(A) tail and one polyadenylation signal AATAAA. The results of protein BLAST in NCBI showed that MnCatL had four active sites, Gln, Cys, His, and Asn, and six S2 subsites, Leu, Met, Ala, Leu, Gly, and Ala, in the mature peptide. The amino acid sequence of MnCatL contains an ERFNIN motif (single-overlined in Figure 2) and an imperfect GNFD motif (double-overlined in Figure 2). 
1 ACGCGGGGAGTTCAGGGTGACAGTCAGCAAGATGAAGTACCTCTGTGCATTAGTTCTTATAGCAGTGGCGGCTTCG

$$
\begin{array}{lllllllllllllll}
M & K & Y & L & C & A & L & V & L & I & A & V & A & A & S
\end{array}
$$

77 GCCTCAGCCGTTTCCTTCTTCACTGTGGTAATGGAGGAGTGGGAGAGTTTCAAGTTTGAACATAGTAAAAAATAT \begin{tabular}{lllllllllllllllllllllllll}
$A$ & $S$ & $A$ & $\mathrm{~V}$ & $\mathrm{~S}$ & $\mathrm{~F}$ & $\mathrm{~F}$ & $\mathrm{~T}$ & $\mathrm{~V}$ & $\mathrm{~V}$ & $\mathrm{M}$ & $\mathrm{E}$ & $\mathrm{E}$ & $\mathrm{W}$ & $\mathrm{E}$ & $\mathrm{S}$ & $\mathrm{F}$ & $\mathrm{K}$ & $\mathrm{F}$ & $\mathrm{E}$ & $\mathrm{H}$ & $\mathrm{S}$ & $\mathrm{K}$ & $\mathrm{K}$ & $\mathrm{Y}$ \\
\hline
\end{tabular} 152 GAAAGTGATACCGAAGAAACATTCCGTATGAAGATCTTTGCTGAGAATAAGCAGAAAATTGCTGCCCACAACAAG \begin{tabular}{lllllllllllllllllllllllll} 
E & $\mathrm{S}$ & $\mathrm{D}$ & $\mathrm{T}$ & $\mathrm{E}$ & $\mathrm{E}$ & $\mathrm{T}$ & $\mathrm{F}$ & $\mathrm{R}$ & $\mathrm{M}$ & $\mathrm{K}$ & $\mathrm{I}$ & $\mathrm{F}$ & $\mathrm{A}$ & $\mathrm{E}$ & $\mathrm{N}$ & $\mathrm{K}$ & $\mathrm{Q}$ & $\mathrm{K}$ & $\mathrm{I}$ & $\mathrm{A}$ & $\mathrm{A}$ & $\mathrm{H}$ & $\mathrm{N}$ & $\mathrm{K}$ \\
\hline
\end{tabular} 227 CTCTATCATACTGGTTCAAAGACTTACAAACTTGGGATGAACAAATATGGTGATATGCTTCATCACGAATTTGTG \begin{tabular}{lllllllllllllllllllllllll}
$\mathrm{L}$ & $\mathrm{Y}$ & $\mathrm{H}$ & $\mathrm{T}$ & $\mathrm{G}$ & $\mathrm{S}$ & $\mathrm{K}$ & $\mathrm{T}$ & $\mathrm{Y}$ & $\mathrm{K}$ & $\mathrm{L}$ & $\mathrm{G}$ & $\mathrm{M}$ & $\mathrm{N}$ & $\mathrm{K}$ & $\mathrm{Y}$ & $\mathrm{G}$ & $\mathrm{D}$ & $\mathrm{M}$ & $\mathrm{L}$ & $\mathrm{H}$ & $\mathrm{H}$ & $\mathrm{E}$ & $\mathrm{F}$ & $\mathrm{V}$ \\
\hline
\end{tabular} 302 AACATGATGAATGGATTCCGTGCTAACACCAGTGGTGCTGGTTACAAGGCAAACCGTGGTTTTCAAGGAGCACAC \begin{tabular}{llllllllllllllllllllllllll}
$N$ & $M$ & $M$ & $N$ & $G$ & $F$ & $R$ & $A$ & $N$ & $T$ & $S$ & $G$ & $A$ & $G$ & $Y$ & $K$ & $A$ & $N$ & $R$ & $G$ & $F$ & $Q$ & $G$ & $A$ & $H$ \\
\hline
\end{tabular} 377 TTTGTGGAGCCACCTGAGGATGTTGTAATGCCCAAAAGTGTTGACTGGAGGGAGAAAGGAGCAGTCACTGAAGTA

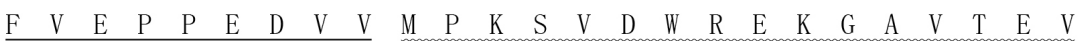
452 AAGGACCAGGGATCGTGTGGCTCATGTTGGGCTTTTTCTGCAACTGGAGCTTTGGAAGGTCAGCATTACAGACAA

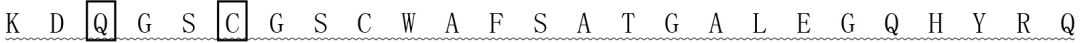
527 ACTGGAGACTTGGTAAGCCTCTCTGAACAAAACTTGGTCGATTGCTCTTCTAAGTTCGGAAATAATGGTTGCAAT

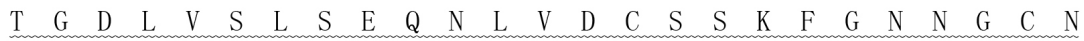
602 GGTGGTCTCATGGACAATGCATTCCAGTACATCAAAGTCAATGGTGGCATTGACACTGAAAAAAGTTACCCTTAT

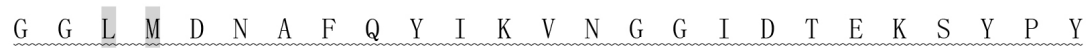
677 GAAGCAGAGGACGAACCATGCCGGTACAATCCTGCAAATGCTGGTGCTGATGATAGGGGCTTTGTGGATGTCCGT $\begin{array}{lllllllllllllllllllllllll}E & A & E & D & E & P & C & R & Y & N & P & A & N & A & G & A & D & D & R & G & F & V & D & V & R\end{array}$ 752 GAAGGAAACGAAAATGCTTTGAAGAAAGCCATTGCCACAATTGGGCCGGTTTCTGTTGCTATCGATGCTAGCCAA

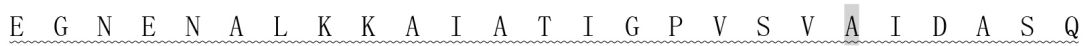
827 GACTCCTTCCAGTTCTATCAACATGGAGTATACAGTGATCCAGACTGCTCAGCAG AAAATTTGGATCATGGAGTC

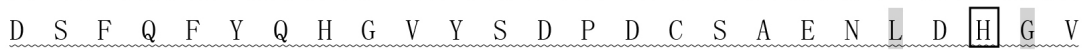
902 CTTGCTGTTGGCTATGGCACTACTGAGGATGGCCAAGACTACTGGCTTGTGAAGAACTCGTGGAGCAAGTCATGG

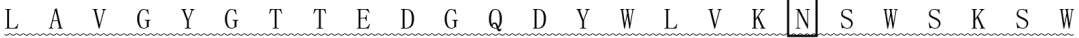
977 GGTGACCAAGGCTACATTAAGATTGCACGCAATCAAAACAATATGTGCGGCATCGCAAGTGCTGCATCCTATCCC

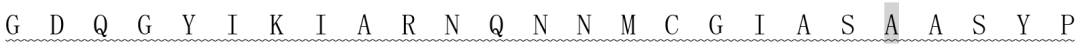
1052 TTGGTTTAAATGTCAGCCTTGTTTGAATTTTCAACATCTTAAACTTCCTCACTAGTGGTATGTTCAGGCCAAGTT $\mathrm{L} \quad \mathrm{V} *$

1127 AGACTGTTATACTCT TTCATTAAGACCGGAAGAGAAAAATTAAAAAGAGGAGTTAATACCTGGTGGTTATGAATT 1202 ATTTTACAACTGTCAACACTTTCAATAAGTGTTTTTTTTAATACAAAATTGAAGTGATTAGTGTTAAGGAAATGA 1277 GCATTATGTAGTTAAAGTAGCATTTACTTGTTAAATGTGACTGGAGCAGTAGATTTTAGGAGACATTGAAAATTG 1352 GTTTTTATCAGATGCTTTTATGGTTTTTAATAGAAATTCAATACTATTCTAAATGTGATA GGTTGTGATTTTATA 1427 TTCTTGATTAATGGGAACAGCTTACTGGCCGGAGAGCTTAGTCTGTGATTCATAGATTTTTATAATGTTTATATG 1502 ACCATTTCAGATATGGTTCAGTGTAATTTCTTTGTTTAATGTACTATTTCAGCTTGTAAGGTTTGCATCTATGAA 1577 TTTCCCAGATCCCAGTTTATATTTACACTT TCATAATTTGTTTATAAACAATTACACTTGGAATTAAAAAAATGT 1652 ATAGATGTGCTGCACAATTTTTA $\underline{\text { AATAAATACTTAAAAAAAAAAAAAAAAAAAAAAAA1710 }}$

Figure 1. Complete cDNA sequence and deduced amino acid sequence of MnCatL. The sequence of the signal peptide is shown in italics at the N-terminus. The propeptide inhibitor domain is underlined. Mature polypeptide is wavylined. The active sites are boxed. The S2 subsites are shaded. The polyadenylation signals are underlined in bold italics. 

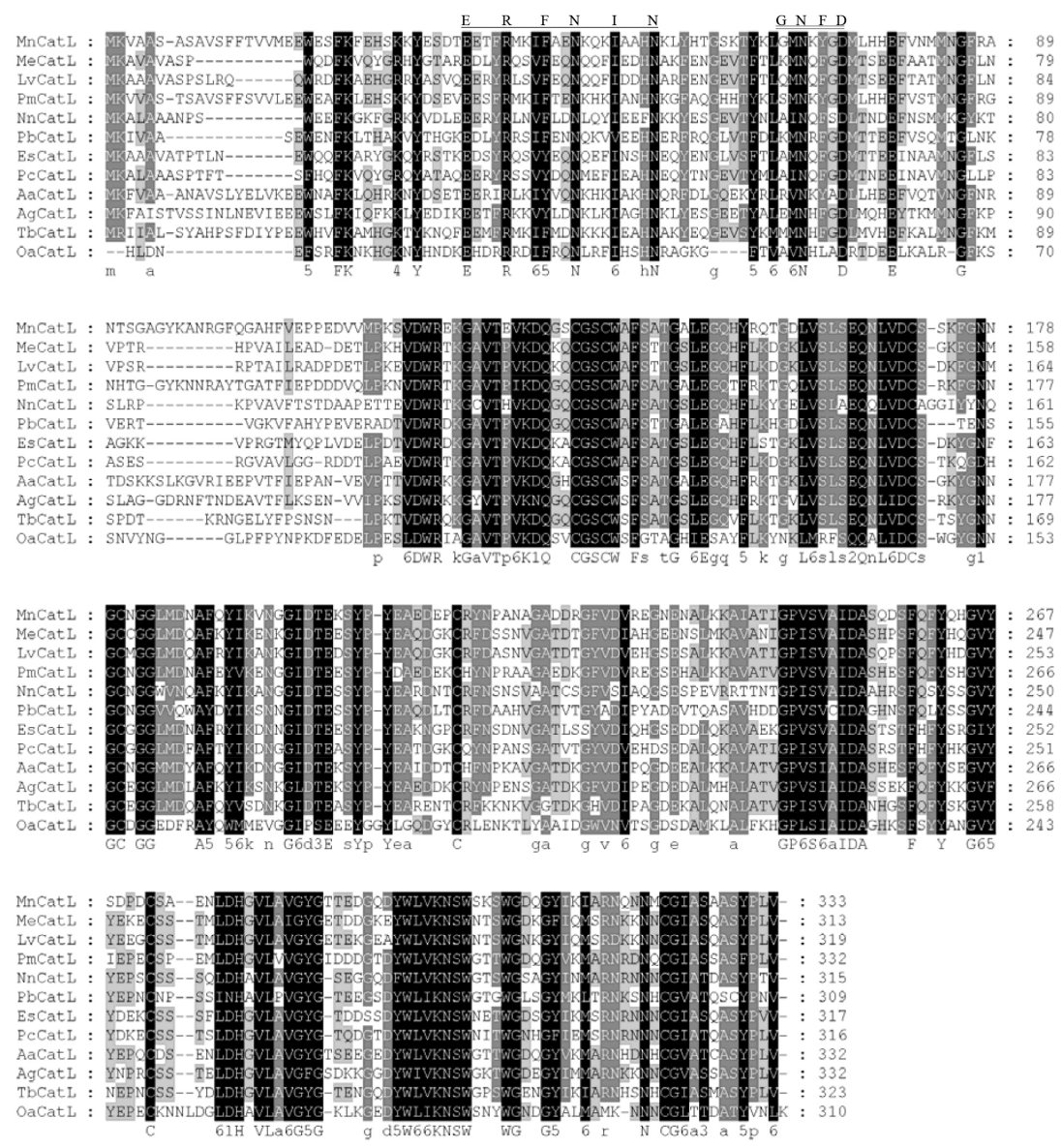

Figure 2. Alignment homologues of MnCatL with other arthropods. The shaded regions indicate identical residues. Other conserved but not consensus amino acids are shaded in gray. The ERFNIN motif is a single overline. The GNFD motif is with double overlines. The organisms and their cathepsin L gene No. include Metapenaeus ensis (AAM96000), Litopenaeus vannamei (CAA68066), Penaeus monodon (ABQ10739), Nephrops norvegicus (CAA56914), Pandalus borealis (BAC65418), Eriocheir sinensis (ADO65980), Paralithodes camtschaticus (ADQ73946), Aedes aegypti (ABE72970), Aphis gossypii (CAD33266), Triatoma brasiliensis (ACF48469), and Ochlerotatus atropalpus (ABE72974).

\section{Distribution of MnCatL mRNA in different tissues}

The mRNA transcripts of MnCatL were widely detected in all tissues examined (Figure 3A). The highest MnCatL expression was observed in the female hepatopancreas (HPF) and HPM. MnCatL was moderately expressed in the muscle, heart, thoracic ganglia, gills, intestine, ovary, and testis, while the lowest was in the female hemocytes (HAF) and HAM. There were significant differences in MnCatL expression between HPM and HPF and between other tissues $(\mathrm{P}<0.05)$. MnCatL expression in HAF and HAM was significantly lower than in other tissues. 
A

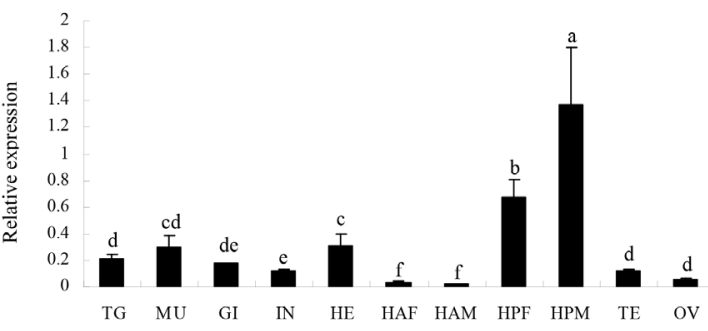

B

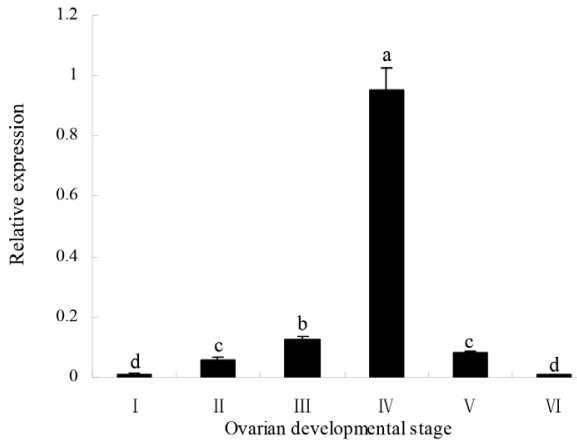

C

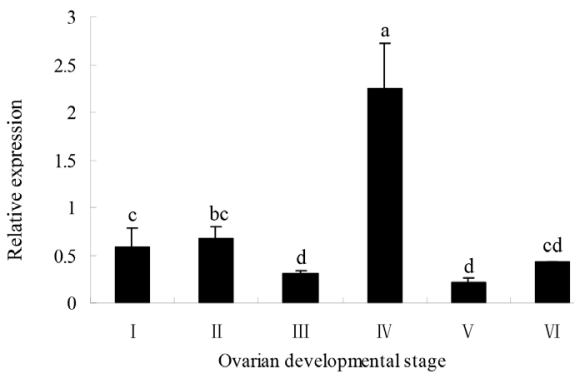

D

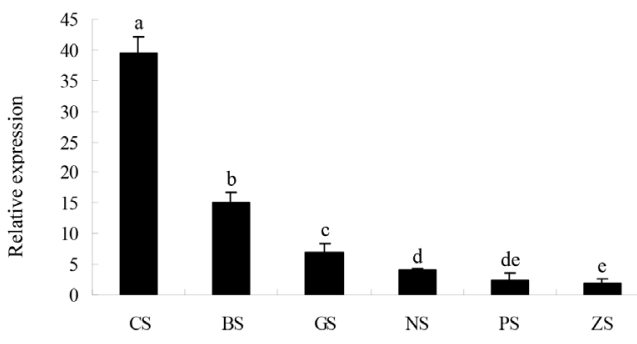

Figure 3. MnCatL mRNA expressions in various tissues of the oriental river prawn. A. $\mathrm{TG}=\mathrm{MnCatL}$ transcripts in the thoracic ganglia; $\mathrm{MU}=$ muscle; $\mathrm{GI}=$ gill; $\mathrm{IN}=$ intestine; $\mathrm{HE}=$ heart; $\mathrm{HAF}=$ female hemocytes; $\mathrm{HAM}=$ male hemocytes; $\mathrm{HPF}=$ female hepatopancreas; $\mathrm{HPM}=$ male hepatopancreas; $\mathrm{TE}=$ testis and $\mathrm{OV}=$ ovary. $\mathbf{B} . \mathrm{MnCatL}$ mRNA expression in ovaries during ovarian maturation stages I (previtellogenesis early stage), II (previtellogenesis later stage), III (vitellogenesis early stage), IV (vitellogenesis later stage), V (mature stage), VI (paracmasis stage). C. MnCatL mRNA expression in the hepatopancreas during ovarian maturation. D. MnCatL mRNA expression in different developmental stages of the embryo: $\mathrm{CS}=$ cleavage stage; $\mathrm{BS}=$ blastula stage; GS = gastrula stage; NS $=$ nauplius stage; $\mathrm{PS}=$ protozoea stage $\mathrm{ZS}=$ zoea stage. Values are reported as means $\pm \mathrm{SE}$. Values with different superscripts are significantly different $(\mathrm{P}<0.05)$. 


\section{MnCatL expression during oogenesis and embryogenesis}

The expression of MnCatL in the ovary and hepatopancreas during oogenesis was measured (Figure 3B and C). MnCatL expression changed between ovarian stages. MnCatL expression in ovary gradually increased from the early stage of previtellogenesis (stage I) to the early stage of vitellogenesis (stage III) and quickly reached a maximum at the late stage of vitellogenesis (stage IV), then falling in the late stage. Transcripts of MnCatL in the hepatopancreas peaked at ovarian stage IV while the transcript difference at other stages was not significant. MnCatL expression in hepatopancreas was higher than that in the ovary. The expression of MnCatL in embryos is shown in Figure 3D. The expression of MnCatL significantly decreased during embryonic development. MnCatL expression decreased drastically from the cleavage stage to the gastrula stage, while from the nauplius stage to the protozoea stage, the expression change was not significant $(\mathrm{P}>0.05)$.

\section{DISCUSSION}

CatL cDNA has been identified in various economically important aquatic crustacean species, including American lobster (Homarus americanus) (Laycock et al., 1992), shrimp M. ensis (Hu and Leung, 2004), Penaeus vannamei (now named Litopenaeus vannamei) (Liu and Warner, 2006) and Chinese crab (Eriocheir sinensis) (Li et al., 2010). CatL cDNAs were also cloned in Norway lobster (Nephrops norvegicus) (Le Boulay et al., 1996), northern shrimp (Pandalus borealis) (Aoki et al., 2004) and brine shrimp (Artemia franciscana) (Liu and Warner, 2006). However, there is little information on CatL cDNA in freshwater prawn species. This is the first report on the gene cloning and expression of CatL in a freshwater prawn.

The CatL family propeptides contain two signature motifs: a highly conserved interspersed ER(F/W)NIN motif, which can inhibit proteolytic activity in the family of cysteine proteinases (Karrer et al., 1993), and a GNFD motif, which is involved in intramolecular processing in propapain (Vernet et al., 1995). The alignment homologues result showed that the base sequence of MnCatL was homologous to CatL in GenBank and that MnCatL comprised an ERFNIN and an imperfect GNFD motif. The presence of the ER(F/W)NIN and GNFD motifs in the region of MnCatL clearly indicates its relationship to the CatL group.

The amino acid polypeptide of MnCatL consisted of a signal peptide, a propeptide and a mature peptide. Like other members in the papain family, CatL is also synthesized as precursor molecules containing a signal peptide, an activation propeptide and a catalytically active mature domain (Tao et al., 1994). CatL is an inactive precursor and only becomes activated after proteolytic removal of the N-terminal propeptide (Rawlings and Barrett, 1994). The signal peptide, propeptide and mature peptide of MnCatL consisted of 18, 106 and 218 amino acids, respectively. This result is within the range of previous reports since CatL is composed of a 10- to 20 -amino acid signal peptide, a 36- to 315-amino acid propeptide and a 220- to 260-amino acid mature peptide (McGrath, 1999; Turk et al., 2000; Lecaille et al., 2002). The only exception is that the mature peptide of MnCatL in this study was two amino acids fewer than that reported earlier. Similar results were observed in other crustaceans, since the CatL mature peptide in Penaeus monodon (ABQ10739.1), Artemia franciscana (AAV63977.1) and A. salina (ABS17682.1) are 215, 214 and 214 amino acids, respectively. On the other hand, the mature cysteine protease of Dermestes frischii contains 219-amino acid residues (Papisova et al., 2011). 
Four active sites, Gln, Cys, His, and Asn, were found in the amino acid sequence of MnCatL. CatL belongs to the $\mathrm{C} 1$ family peptidases whose catalytic residues are Cys and His, forming a catalytic dyad. The Gln and Asn residues play an important role in catalysis. Gln preceding the catalytic Cys is believed to help the formation of the oxyanion hole (Papisova et al., 2011). The Asn residue orients the imidazolium ring of the catalytic His. Besides the catalytic triad residue Cys, six additional cysteine residues were found in MnCatL to form three disulfide bridges in papain (Kamphuis et al., 1984). The same phenomenon was reported in the CatL of Pandulus borealis (Aoki et al., 2004). MnCatL had six S2 subsites, which are the dominant substrate specificity subsites of papain-like cysteine proteases (McGrath, 1999; Turk et al., 2000).

MnCatL was detected in all tissues tested, including hepatopancreas, ovary, muscle, intestine, gill, thoracic ganglia, heart, testis, and hemocytes in the oriental river prawn, which is consistent with the ubiquitous expression in tissues of other animals (Brix et al., 2008; Li et al., 2010). CatL plays a housekeeping role in crustaceans (Hu and Leung, 2004) and we found the highest expression of MnCatL in the hepatopancreas. The high expression of MnCatL in the hepatopancreas was also reported in M. ensis (Hu and Leung, 2004) and E. sinensis (Li et al., 2010). The hepatopancreas is responsible for the synthesis of many digestive enzymes in decapods (Gibson and Barker, 1979). Therefore, the predominant expression of CatL in the hepatopancreas of oriental river prawn suggests its involvement in food digestion, since the association of CatL with food digestion has been found in other decapods such as American lobster H. americanus (Laycock et al., 1992) and Norway lobster $N$. norvegicus (Le Boulay et al., 1996). The evidence at the cellular level also shows that the CatL is localized in large digestive vacuoles of the digestive B cell of hepatopancreas in M. ensis (Hu and Leung, 2004).

MnCatL was not only present in the hepatopancreas but also in the ovary in oriental river prawn. Its expressions in the ovary and hepatopancreas changed as ovary development progressed. In our previous study, ovary and hepatopancreas were found to be the source of VTG in oriental river prawns. Therefore, these results imply that the roles of MnCatL in the digestive gland and ovary together contribute to reproduction during ovary development in the oriental river prawn. At the same ovary developmental stages, MnCatL expression in the hepatopancreas was higher than that in the ovary, which is consistent with our previous report that the hepatopancreas is the main VTG source organ in oriental river prawns (Zhao, 2010). The level of MnCatL expression was maximal in the later vitellogenesis stage (stage IV) when VTG reached the highest level during ovary development (Zhao, 2010). It seems that MnCatL in both hepatopancreas and ovary is involved with the metabolism of VTG in oriental river prawn. The involvement of CatL has been found in the ovary of other arthropods. For instance, CatL exists in the nucleus of oocyte in M. ensis (Hu and Leung, 2004). Similar results were found in S. zeamais (Matsumoto et al., 1997) and Bombys mor L. (Yarygin et al., 2003). A confirmed function of CatL in the ovary is its involvement in VTG hydrolysis. The role of CatL in VTG hydrolysis has been widely studied in vertebrates (Carnevali et al., 1999, 2006), while its research in arthropods is behind, although some progress has been made in the silkworm $B$. mori (Kageyama and Takahashi, 1990) and soft tick Orithodors moubata (Fagotto, 1990a,b).

In the present study, MnCatL expression was reduced as embryo development progressed in oriental river prawns. Its expression was highest at the cleavage stage and it decreased significantly from the cleavage stage to the gastrula stage while the difference was not significant from the nauplius stage to the zoea stage. The change in expression was related 
to the volume of the yolk sac in the embryo. The yolk sac supplies nutrition for embryo development and the yolk is almost all absorbed in the gastrula stage in oriental river prawns (Zhao, 2005). The result showed that the MnCatL was possibly involved in hydrolyzing yolk protein. This deduction is supported by results reported for other arthropods that the cysteine enzyme purified from silkworm eggs can hydrolyze VTG, vitellin and lipophorin (Kageyama and Takahashi, 1990), and that CatL is stored in the yolk to degrade VTG in the soft tick (Fagotto, 1990a,b). The expression of MnCatL during oogenesis and embryogenesis in oriental river prawns implies that CatL can functionally degrade VTG and yolk proteins.

\section{ACKNOWLEDGMENTS}

Research supported by the Chinese Natural Science Foundation (\#31101887, \#31172422 and \#31001098), the Shanghai Committee of Science and Technology, China (\#10JC1404100 and \#09ZR1409800), the Jiangsu Province Natural Science Foundation (\#BK2011419), the National Basic Research Program (“973” Program, \#2009CB118702), the Shanghai Technology System for Chinese mitten-handed crab, Special Fund for AgroScientific Research in the Public Interest (\#201003020 and \#201203065), the National "Twelfth Five-Year" Plan for Science \& Technology Support (\#2012BAD25B03), the Jiangsu Province aquaculture "Three projects" (\#PJ2010-59), and partly by the E-institute of the Shanghai Municipal Education Commission (\#E03009).

\section{REFERENCES}

Aoki H, Ahsan MN and Watabe S (2004). Molecular and enzymatic properties of a cathepsin L-like proteinase with distinct substrate specificity from northern shrimp (Pandalus borealis). J. Comp. Physiol. B 174: 59-69.

Brix K, Dunkhorst A, Mayer K and Jordans S (2008). Cysteine cathepsins: cellular roadmap to different functions. Biochimie 90: 194-207.

Carnevali O, Carletta R, Cambi A, Vita A, et al. (1999). Yolk formation and degradation during oocyte maturation in seabream Sparus aurata: involvement of two lysosomal proteinases. Biol. Reprod. 60: 140-146.

Carnevali O, Cionna C, Tosti L, Lubzens E, et al. (2006). Role of cathepsins in ovarian follicle growth and maturation. Gen. Comp Endocrinol. 146: 195-203.

Chen JS, Sappington TW and Raikhel AS (1997). Extensive sequence conservation among insect, nematode, and vertebrate vitellogenins reveals ancient common ancestry. J. Mol. Evol. 44: 440-451.

Fagotto F (1990a). Yolk degradation in tick eggs: I. Occurrence of a cathepsin L-like acid proteinase in yolk spheres. Arch. Insect Biochem. Physiol. 14: 217-235.

Fagotto F (1990b). Yolk degradation in tick eggs: II. Evidence that cathepsin L-like proteinase is stored as a latent, acidactivable proenzyme. Arch. Insect Biochem. Physiol. 14: 237-252.

Fagotto F and Maxfield FR (1994). Yolk platelets in Xenopus oocytes maintain an acidic internal pH which may be essential for sodium accumulation. J. Cell Biol. 125: 1047-1056.

Gibson R and Barker PL (1979). The decapod hepatopancreas. Oceanogr. Mar. Biol. Ann. Rev. 17: 285-346.

$\mathrm{Hu}$ KJ and Leung PC (2004). Shrimp cathepsin L encoded by an intronless gene has predominant expression in hepatopancreas, and occurs in the nucleus of oocyte. Comp Biochem. Physiol. B Biochem. Mol. Biol. 137: 21-33.

$\mathrm{Hu} \mathrm{KJ}$ and Leung PC (2007). Food digestion by cathepsin L and digestion-related rapid cell differentiation in shrimp hepatopancreas. Comp Biochem. Physiol. B Biochem. Mol. Biol. 146: 69-80.

Kageyama T and Takahashi SY (1990). Purification and characterization of a cysteine proteinase from silkworm eggs. Eur. J. Biochem. 193: 203-210.

Kamphuis IG, Kalk KH, Swarte MB and Drenth J (1984). Structure of papain refined at 1.65 Å resolution. J. Mol. Biol. 179: 233-256.

Karrer KM, Peiffer SL and DiTomas ME (1993). Two distinct gene subfamilies within the family of cysteine protease genes. Proc. Natl. Acad. Sci. U. S. A. 90: 3063-3067. 
Kwon JY, Prat F, Randall C and Tyler CR (2001). Molecular characterization of putative yolk processing enzymes and their expression during oogenesis and embryogenesis in rainbow trout (Oncorhynchus mykiss). Biol. Reprod. 65: 1701-1709.

Laycock MV, MacKay RM, Di Fruscio M and Gallant JW (1992). Molecular cloning of three cDNAs that encode cysteine proteinases in the digestive gland of the American lobster (Homarus americanus). FEBS Lett. 301: 125.

Le Boulay C, Van Wormhoudt A and Sellos D (1996). Cloning and expression of cathepsin L-like proteinases in the hepatopancreas of the shrimp Penaeus vannamei during the intermolt cycle. J. Comp Physiol. B 166: 310-318.

Lecaille F, Choe Y, Brandt W, Li Z, et al. (2002). Selective inhibition of the collagenolytic activity of human cathepsin K by altering its $\mathrm{S} 2$ subsite specificity. Biochemistry $41: 8447-8454$.

Li WW, Jin XK, He L, Jiang H, et al. (2010). Molecular cloning, characterization, expression and activity analysis of cathepsin L in Chinese mitten crab, Eriocheir sinensis. Fish Shellfish Immunol. 29: 1010-1018.

Liu L and Warner AH (2006). Further characterization of the cathepsin L-associated protein and its gene in two species of the brine shrimp, Artemia. Comp Biochem. Physiol. A Mol. Integr. Physiol. 145: 458-467.

Matsumoto I, Emori Y, Abe K and Arai S (1997). Characterization of a gene family encoding cysteine proteinases of Sitophilus zeamais (maize weevil), and analysis of the protein distribution in various tissues including alimentary tract and germ cells. J. Biochem. 121: 464-476.

McGrath ME (1999). The lysosomal cysteine proteases. Annu. Rev. Biophys. Biomol. Struct. 28: 181-204.

Meusy JJ and Payen GG (1988). Female reproduction in Malacostracan Crustacea. Zool. Sci. 5: 217-265.

Papisova AI, Javadov A, Rudenskaya YA, Balandina GN, et al. (2011). Novel cathepsin L-like protease from dermestid beetle Dermestes frischii maggot. Biochimie 93: 141-148.

Rawlings ND and Barrett AJ (1994). Families of cysteine peptidases. Methods Enzymol. 244: 461-486.

Ren Q, Zhang XW, Sun YD, Sun SS, et al. (2010). Two cysteine proteinases respond to bacterial and WSSV challenge in Chinese white shrimp Fenneropenaeus chinensis. Fish Shellfish Immunol. 29: 551-556.

Soares-Costa A, Dias AB, Dellamano M, de Paula FF, et al. (2011). Digestive physiology and characterization of digestive cathepsin L-like proteinase from the sugarcane weevil Sphenophorus levis. J. Insect Physiol. 57: 462-468.

Tao K, Stearns NA, Dong J, Wu QL, et al. (1994). The proregion of cathepsin L is required for proper folding, stability, and ER exit. Arch. Biochem. Biophys. 311: 19-27.

Turk B, Turk D and Turk V (2000). Lysosomal cysteine proteases: more than scavengers. Biochim. Biophys. Acta 1477: 98-111.

Vernet T, Berti PJ, de Montigny C, Musil R, et al. (1995). Processing of the papain precursor. The ionization state of a conserved amino acid motif within the Pro region participates in the regulation of intramolecular processing. J. Biol. Chem. 270: 10838-10846.

Wallace RA (1985). Vitellogenesis and Oocyte Growth in Non-Mammalian Vertebrates. In: Developmental Biology (Browder LW, ed.). Plenum, New York, 127-177.

Wu P, Qi D, Chen L, Zhang H, et al. (2009). Gene discovery from an ovary cDNA library of oriental river prawn Macrobrachium nipponense by ESTs annotation. Comp. Biochem. Physiol. Part D Genomics Proteomics 4: 111-120.

Yamamoto Y, Takimoto K, Izumi S, Toriyama-Sakurai M, et al. (1994). Molecular cloning and sequencing of cDNA that encodes cysteine proteinase in the eggs of the silkmoth, Bombyx mori. J. Biochem. 116: 1330-1335.

Yarygin DV, Klunova SM and Filippovich YB (2003). Isolation, purification, and properties of cysteine proteinase from Bombyx mori L. eggs. Biochemistry 68: 63-67.

Zhang F, Chen L, Wu P, Zhao W, et al. (2010a). cDNA cloning and expression of Ubc9 in the developing embryo and ovary of Oriental river prawn, Macrobrachium nipponense. Comp Biochem. Physiol. B Biochem. Mol. Biol. 155: 288-293.

Zhang J, Li F, Jiang H, Yu Y, et al. (2010b). Proteomic analysis of differentially expressed proteins in lymphoid organ of Fenneropenaeus chinensis response to Vibrio anguillarum stimulation. Fish Shellfish Immunol. 29: 186-194.

Zhao W (2010). Cloning and Expression of the Genes Involving in the Vitellogenin Metabolism in Oriental River Prawn, Macrobrachium nipponense. East China Normal University, Shanghai.

Zhao W, Chen L, Qin J, Wu P, et al. (2011). MnHSP90 cDNA characterization and its expression during the ovary development in oriental river prawn, Macrobrachium nipponense. Mol. Biol. Rep. 38: 1399-1406.

Zhao Y (2005). Studies on Embryonic Development in Freshwater Prawn Macrobrachium nipponense (Decapoda, Palaemonidae). East China Normal University, Shanghai. 